IFUP-TH 38/93

September 1993

hep-th/9309034

\title{
The algebraic structure of the generalized uncertainty principle
}

\author{
Michele Maggiore \\ I.N.F.N. and Dipartimento di Fisica dell'Università, \\ piazza Torricelli 2, I-56100 Pisa, Italy.
}

\begin{abstract}
We show that a deformation of the Heisenberg algebra which depends on a dimensionful parameter $\kappa$ is the algebraic structure which underlies the generalized uncertainty principle in quantum gravity. The deformed algebra and therefore the form of the generalized uncertainty principle are fixed uniquely by rather simple assumptions. The string theory result is reproduced expanding our result at first order in $\Delta p / M_{\mathrm{PL}}$. We also briefly comment on possible implications for Lorentz invariance at the Planck scale.
\end{abstract}


In recent years it has been suggested that measurements in quantum gravity are governed by a generalized uncertainty principle

$$
\Delta x \geq \frac{\hbar}{\Delta p}+\text { const. } G \Delta p
$$

( $G$ is Newton's constant). At energies much below the Planck mass $M_{\mathrm{PL}}$ the extra term in eq. (1) is irrelevant and the Heisenberg relation is recovered. As we approach the Planck mass, this term becomes important and it is responsible for the existence of a minimal observable length on the order of the Planck length.

The result (1) was first suggested in the context of string theory [1-4], in the kinematical region where $2 G E$ is smaller than the string length (for a review, see [5]). However, heuristic arguments [6] suggest that this formula might have a more general validity in quantum gravity, and it is not necessarily related to strings. It is therefore natural to ask whether there is an algebraic structure which reproduces eq. (1) (or, more in general, which reproduces the existence of a minimal observable length), in the same way in which the Heisenberg uncertainty principle follows from the algebra $[x, p]=i \hbar$.

In this Letter we answer (in the affirmative) this question. Our strategy is as follows. Since it is relatively clear that no Lie algebra can reproduce eq. (1), we turn our attention to deformed algebras. A deformed algebra is an associative algebra where it is defined a commutator which is non-linear in the elements of the algebra; and there is a deformation parameter such that, in an appropriate limit, a Lie algebra is recovered.

We therefore look for the most general deformed algebra円 which can be constructed from coordinates $x_{i}$ and momenta $p_{i}(i=1,2,3)$. We restrict the range of possibilities making the following assumptions. 1) The threedimensional rotation group is not deformed; the angular momentum $\mathbf{J}$ satisfies the undeformed $S U(2)$ commutation relations, and coordinate and momenta satisfy the undeformed commutation relations $\left[J_{i}, x_{j}\right]=i \epsilon_{i j k} x_{k},\left[J_{i}, p_{j}\right]=$ $i \epsilon_{i j k} p_{k}$. 2) The momenta commutes between themselves: $\left[p_{i}, p_{j}\right]=0$, so that also the translation group is not deformed. 3) The $[x, x]$ and $[x, p]$ commutators depend on a deformation parameter $\kappa$ with dimensions of mass. In the limit $\kappa \rightarrow \infty$ (that is, $\kappa$ much larger than any energy), the canonical commutation relations are recovered.

\footnotetext{
${ }^{1}$ We will not require the existence of a coproduct and of an antipode, which would promote the deformed algebra to a quantum algebra, see below.
} 
Note that we are ready to accept a non-zero commutator between the $x$ 's. While this is at first surprising, we will see that if $\kappa \sim$ Planck mass, the non-commutativity shows up only at the level of the Planck length. The idea is in the spirit of the approach to the structure of spacetime at small distances suggested by non-commutative geometry, pioneered by Connes [0], which underlies much of the applications of quantum groups to gravity. One should also note that our formalism is not Lorentz covariant. We will come back to this important point below.

With these assumptions, the most general form of the $\kappa$-deformed algebra is

$$
\begin{aligned}
{\left[x_{i}, x_{j}\right] } & =\frac{\hbar^{2} a(E)}{\kappa^{2}} i \epsilon_{i j k} J_{k} \\
{\left[x_{i}, p_{j}\right] } & =i \hbar \delta_{i j} f(E) .
\end{aligned}
$$

Here $a(E)$ and $f(E)$ are real, dimensionless functions of $E / \kappa$, and $E^{2}=$ $p^{2}+m^{2}$; the angular momentum $\mathbf{J}$ is defined as dimensionless, so on the right-hand side the dimensions are carried by $\hbar$ and $\kappa$ only. The fact that this is the most general form compatible with our assumptions is clear from the following considerations: the factors of $i$ are determined by the condition of hermiticity of $x_{i}, p_{i}$ and $J_{i}$. The powers of $\hbar$ and $\kappa$ are dictated by dimensional analysis. The tensor $\epsilon_{i j k}$ in eq. (2) appears because we assume that the threedimensional rotation group is undeformed and then it is the only tensor antisymmetric in $i, j$; it must be contracted with $J_{k}$ rather than $p_{k}$ or $x_{k}$ because of parity. One might also add to the right-hand side of eq. (2) a term proportional to $x_{i} p_{j}-x_{j} p_{i}$. (Note that $L_{k}=\epsilon_{i j k}\left(x_{i} p_{j}-x_{j} p_{i}\right)$ cannot be identified with the angular momentum in the spinless case, since it does not satisfy the $S U(2)$ commutation relations unless $f(E)=1$ ). However such a term can be eliminated with a non-local redefinition of coordinates, $\xi_{i}=g(E) x_{i}$, with a suitable function $g(E)$. In the second equation, again $\delta_{i j}$ must appear because it is the only available tensor under rotation. In order to recover the undeformed limit, we further require that $f(0)=1$ and that $a(E)$ is less singular than $E^{-2}$ as $E \rightarrow 0$. We neglect the possibility that the functions $a, f$ depend also on other scalars like $x^{2}$ or $\mathbf{x} \cdot \mathbf{p}$.

Of course, the form of the functions $a(E), f(E)$ is severely restricted by the Jacobi identities. Let us consider first the Jacobi identity $\left[x_{i},\left[x_{j}, x_{k}\right]\right]+$ cyclic $=0$. Using $\left[x_{i}, E\right]=i \hbar f(E) p_{i} / E$, and $\left[x_{i}, a(E)\right]=i \hbar f(E)\left(p_{i} / E\right) d a / d E$, 
we get

$$
\frac{d a}{d E} \mathbf{p} \cdot \mathbf{J}=0
$$

Since the Jacobi identity must be satisfied independently of the particular representation of the algebra, that is independently of whether the condition $\mathbf{p} \cdot \mathbf{J}=0$ holds or not, we conclude that $a(E)=$ const. With a redefinition of $\kappa$ we can set this constant to \pm 1 .

The Jacobi identity $\left[x_{i},\left[x_{j}, p_{k}\right]\right]+$ cyclic $=0$ gives

$$
\frac{f(E)}{E} \frac{d f}{d E}=\mp \frac{1}{\kappa^{2}}
$$

where the upper (lower) sign correspond to the choice $a=+1(-1)$. Since $f(0)=1$, eq. (5) gives $f(E)=\left(1 \mp\left(E^{2} / \kappa^{2}\right)\right)^{1 / 2}$. All other Jacobi identities are automatically satisfied. Of course, to satisfy the Jacobi identities is in general highly non-trivial, especially in a deformed algebra. It is remarkable that in our case $i$ ) there exists a solution: thus, we can $\kappa$-deform the Heisenberg algebra. ii) The solution is unique (within our assumptions and apart from the \pm sign). In the following we only consider the lower sign ${ }^{2}$ and write the $\kappa$-deformed Heisenberg algebra as

$$
\begin{aligned}
{\left[x_{i}, x_{j}\right] } & =-\frac{\hbar^{2}}{\kappa^{2}} i \epsilon_{i j k} J_{k} \\
{\left[x_{i}, p_{j}\right] } & =i \hbar \delta_{i j}\left(1+\frac{E^{2}}{\kappa^{2}}\right)^{1 / 2} .
\end{aligned}
$$

Actually, we already derived eqs. (6,7) in ref. [8], following a rather different route: we considered the $\kappa$-deformed Poincaré algebra suggested in 9] and we discussed how to generalize the Newton-Wigner localization operator to the $\kappa$-deformed case. We then obtained the algebra $(6,7)$, with $x_{i}$ identified with the $\kappa$-deformed Newton-Wigner operator and $p_{i}, J_{i}$ identified with the generators of spatial translations and rotations of the $\kappa$-deformed Poincaré algebra. (To compare with the results of ref. [8] one must observe that the present definition of $\kappa$ differs by a factor of 2 from that used in [9, 8] and that in eq. (7) $E^{2}$ is a shorthand for $\mathbf{p}^{2}+m^{2}$, while in the $\kappa$-Poincaré

\footnotetext{
${ }^{2}$ The solution with the upper sign is however quite intriguing; since $f(E)$ is real by definition, the solution is valid only for $E \leq \kappa$ and describes a system which obeys standard quantum mechanics for $E \ll \kappa$ and satisfies $[x, p]=0$ at $E=\kappa$.
} 
algebra the dispersion relation is, with the present definition of $\kappa, \mathbf{p}^{2}+m^{2}=$ $\left.\kappa^{2} \sinh ^{2}(E / \kappa).\right)$

The derivation that we have presented in this Letter is instead independent of whether we consider the usual or the deformed Poincaré algebra.

We do not attempt to define a coproduct on the deformed algebra given by eqs. $(6,7)$, since a system composed of two particles localized at $x^{(1)}$ and $x^{(2)}$, respectively, cannot be considered as a single localized system and therefore we cannot require, in general, that it can be described by a coordinate $X=X\left(x^{(1)}, x^{(2)}\right)$ which, togheter with the total momentum, satisfies the same algebra as the constituents. Thus, the algebraic structure that we have presented is not a Hopf algebra, nor a quantum algebra.

From eq. (7) we immediately derive the generalized uncertainty principle

$$
\Delta x_{i} \Delta p_{j} \geq \frac{\hbar}{2} \delta_{i j}\left\langle\left(1+\frac{E^{2}}{\kappa^{2}}\right)^{1 / 2}\right\rangle .
$$

Expanding the square root in powers of $(E / \kappa)^{2}$ and using $\left\langle\mathbf{p}^{2}\right\rangle=\mathbf{p}^{2}+(\Delta p)^{2}$, where $(\Delta p)^{2}=\left\langle(\mathbf{p}-\langle\mathbf{p}\rangle)^{2}\right\rangle$, at first order we obtain

$$
\Delta x_{i} \Delta p_{j} \geq \frac{\hbar}{2} \delta_{i j}\left(1+\frac{E^{2}+(\Delta p)^{2}}{2 \kappa^{2}}\right) .
$$

Thus, in the regime $E \ll \kappa, \Delta p \lesssim \kappa$, we recover the string theory result. The constant in eq. (1) is reproduced if we identify $\hbar / \kappa$ with the string length $\lambda_{s}$ times a numerical constant of order one. Note that if $\hbar / \kappa \sim \lambda_{s}$ (and, as usual, the string length is larger than the Planck length), then the condition $E \ll \kappa$ implies the condition $2 G E \ll \lambda_{s}$.

If we consider instead the regime $\langle\mathbf{p}\rangle^{2} \sim(\Delta p)^{2} \gg \kappa^{2}$ we get

$$
\Delta x \geq \text { const } \times \frac{\hbar}{\kappa}
$$

while in the regime $\langle\mathbf{p}\rangle^{2} \gg(\Delta p)^{2},\langle\mathbf{p}\rangle^{2} \gg \kappa^{2}$ we get

$$
\Delta x \geq \text { const } \times \frac{\hbar}{\kappa} \frac{|\langle\mathbf{p}\rangle|}{\Delta p} \gg \frac{\hbar}{\kappa}
$$

It is important to observe that the string theory result, eq. (1), is reproduced in the appropriate kinematical limit after expanding our closed 
expression at first order in $\Delta p / \kappa$. However, only the full expression, eq. (8), has an underlying algebraic origin.

We now comment on the issue of Lorentz invariance. When we discuss the possible existence of a minimal observable length, we actually refer to a spatial length. Such a concept, of course, is not Lorentz invariant. We can always perform a boost and squeeze any 'minimal' length as much as we like. Then, if a minimal spatial length actually exists, we must consider the very interesting possibility that Lorentz invariance is not respected by quantum gravity at a scale on the order of $\hbar / \kappa$. In the formalism of the $\kappa$-deformed Poincaré algebra of [9] this is explicit, since the Lorentz algebra is deformed by the parameter $\kappa$. In our approach, it is implicit in the use of the three vectors $x_{i}, p_{i}$ in place of the corresponding four-vectors. In this context it is interesting to mention that a breaking of Lorentz invariance at the string scale or at the Planck scale has been suggested very recently by Susskind in ref. [10], where it is argued that Lorentz contraction of particles must saturate as $E \sim M_{\mathrm{PL}}$, and a constant value should be approached (cfr. our eqs. $(10,11))$. It might be interesting to observe that the considerations of ref. [10] stem from Gedanken experiments with black holes, and a (different) Gedanken experiment with black holes [6] was also the starting point of our investigation.

Two final comments are in order. The first is that we have a scheme in which Newton's constant $G$ (or equivalently $\kappa$ ) enters the theory at the kinematical level (see also ref. [11] for early attempts in this direction). The minimal length also emerges at a kinematical, rather than dynamical level. This feature is rather satisfying. The second, related comment, is that this formalism can acquire substance only after a suitable dynamics has been added. This is particularly clear if we repeat our analysis in $1+1$ dimensions. In this case only the equation $[x, p]=i \hbar f(E)$ survives. Of course, we can always perform a non-local redefinition of the coordinate, defining $\xi=x / f(E)$, so that $[\xi, p]=i \hbar$. However, one would pay this at the level of the action, which would become non-local. The fact that a non-local particle Lagrangian can generate a minimal observable length has been nicely shown in [12].

I thank M. Mintchev, M. Shifman and A. Vainshtein for useful discussions. 


\section{References}

[1] G. Veneziano, Europhys. Lett. 2 (1986) 199; Proc. of Texas Superstring Workshop (1989).

D. Gross, Proc. of ICHEP, Munich (1988).

[2] D. Amati, M. Ciafaloni and G. Veneziano, Phys. Lett. B197 (1987) 81, B216 (1989) 41; Int. J. Mod. Phys. A3 (1988) 1615; Nucl. Phys. B347 (1990) 530.

[3] D.J. Gross and P.F. Mende, Phys. Lett. B197 (1987) 129; Nucl. Phys. B303 (1988) 407.

[4] K. Konishi, G. Paffuti and P. Provero, Phys. Lett. B234 (1990) 276;

R. Guida, K. Konishi and P. Provero, Mod. Phys. Lett. A6 (1991) 1487.

[5] M. Ciafaloni, preprint DFF 172/9/92.

[6] M. Maggiore, Phys. Lett. B304 (1993) 65.

[7] A. Connes, Non-Commutative Differential Geometry, IHES 62 (1986).

[8] M. Maggiore, preprint IFUP-TH 19/93, hep-th/9305163, May 1993.

[9] J. Lukierski, A. Nowicki and H. Ruegg, Phys. Lett. B293 (1992) 344.

[10] L. Susskind, preprint SU-ITP-93-21, hep-th/9308139, Aug. 1993.

[11] P.K. Townsend, Phys. Rev. D15 (1977) 2795.

[12] M. Kato, Phys. Lett. B245 (1990) 43. 